\title{
Interactions of non-polar and "Click-able" nucleotides in the confines of a DNA polymerase active site $\dagger$
}

\author{
Samra Obeid, ${ }^{* a}$ Holger Bußkamp, ${ }^{a}$ Wolfgang Welte, ${ }^{b}$ Kay Diederichs ${ }^{b}$ and Andreas Marx ${ }^{a}$ \\ Received 11th June 2012, Accepted 25th June 2012 \\ DOI: $10.1039 / \mathrm{c} 2 \mathrm{cc} 34181 \mathrm{f}$
}

Modified nucleotides play a paramount role in many cuttingedge biomolecular techniques. The present structural study highlights the plasticity and flexibility of the active site of a DNA polymerase while incorporating non-polar "Click-able" nucleotide analogs and emphasizes new insights into rational design guidelines for modified nucleotides.

In biological core technologies such as next-generation sequencing, ${ }^{1}$ single molecule sequencing, ${ }^{2}$ microarray analysis based on labeled DNA amplificates, ${ }^{3}$ DNA conjugation, ${ }^{4}$ or the in vitro selection of ligands like aptamers by SELEX (systematic enrichment of ligands by exponential amplification), ${ }^{5}$ functionalized 2'-deoxynucleoside triphosphates (dNTPs) play a fundamental role. One approach to improve the incorporation efficiency of modified substrates is to vary the linkage connecting the nucleotide moiety and the modification of choice. In most cases the modifications were introduced at the nucleobase, in particular, in the case of pyrimidines at the C5 position. ${ }^{1-6}$ For rational linkage design knowledge of the structure-function relationship of the enzyme is of great importance. Several recently solved structures of KlenTaq (the large fragment of Thermus aquaticus DNA polymerase I) caught while processing modified dNTPs were reported. ${ }^{7}$ These studies focused on flexible and polar modifications that have distinct hydrogen-bonding capability. It turned out that the polar functionalities allow proper substrate-protein interaction and thereby might improve the substrate properties. However, little is known about rigid and nonpolar modification. So far, only one DNA polymerase structure capturing a rigid and nonpolar modification is available. ${ }^{7 a}$ This study suggested that the rigid and non-polar modification results in the distortion of the interaction between the enzyme and the substrate leading to the observed low incorporation efficiency. However, in some cases rigid linkers are needed to enable favorable spectroscopic properties. Therefore, we extended the functional and structural studies with a new set of rigid C5

\footnotetext{
${ }^{a}$ Department of Chemistry and Konstanz Research School Chemical Biology, University of Konstanz, Universitätsstrasse 10, 78457 Konstanz, Germany.E-mail: andreas.marx@uni-konstanz.de; Fax: + 49 7531-88-5140; Tel: + 49 7531-88-5139

${ }^{b}$ Department of Biology, University of Konstanz,

Universitätsstrasse 10, 78457 Konstanz, Germany

$\uparrow$ Electronic supplementary information (ESI) available: See DOI: $10.1039 / \mathrm{c} 2 \mathrm{cc} 34181 \mathrm{f}$
}

modified nucleotides (Fig. 1). The introduction of an aromatic ring in the rigid linkage should enable interactions with the protein. Further, these modified nucleotides serve as precursors, which can be post-synthetically addressed by $\mathrm{Cu}(\mathrm{I})$-catalyzed azide-alkyne cycloaddition ("Click" chemistry), due to the terminal alkyne function. ${ }^{6 l, 8} \mathrm{We}$ present crystal structures of KlenTaq caught during the incorporation process of modified nucleotides. The structures reveal that the interaction pattern of the aromatic ring with the protein might account for the efficient processing of the analogs by KlenTaq.

Starting from 5-iodo $2^{\prime}$-deoxyuridine the modification was introduced by palladium-catalyzed cross-coupling ${ }^{6 n, 9}$ with 1,4-diethynylbenzene. Next the modified thymidine analogs were transformed into the corresponding cytidine analogs, ${ }^{10}$ followed by the conversion into the respective thymidine- or cytidine triphosphate analogs ${ }^{11}$ (for details see ESI $\dagger$ ).

Having the modified pyrimidine analogs in hand their acceptance and incorporation efficiency compared to the natural substrates by KlenTaq were investigated by competition experiments ${ }^{3 c, 7}$ (Fig. 1a and b). In these single nucleotide incorporation experiments the modified substrates directly compete with the natural counterpart for incorporation. The objective was to determine the relative ratio of the modified versus the natural substrate that results in equal incorporation efficiencies. This ratio was assigned using $20 \%$ denaturing polyacrylamide gel electrophoresis exploiting the different retention times of the reaction products, due to the additional bulk of the modification. ${ }^{6 n, 7 a, 12}$ The quantification of these experiments revealed that KlenTaq incorporates $\mathrm{dT}^{*} \mathrm{MP}$ and $\mathrm{dC}^{*} \mathrm{MP}$ with only 7- and 2-fold lower efficiency compared to the natural counterparts, respectively (Fig. S1, ESI $\dagger$ ).

Encouraged by the excellent acceptance of the modified substrates, we next investigated the access of the terminal alkyne group via Click reaction (Fig. 1c; Fig. S2 and Scheme S2, ESI $\dagger$ ). First, we performed primer extension experiments using modified substrates to introduce a terminal alkyne group into DNA (Fig. 1c, lane 4). The mono-alkyne functionalized DNA product was subsequently used for the Click reaction. An azido-modified biotin was clicked to the modified DNA. After $4 \mathrm{~h}$ reaction time a quantitative conversion to the biotin labeled DNA product is observed, which shows a slight decrease in retention time, due to the additional bulk of the introduced modification (Fig. 1c, lane 6). Further, incubation with streptavidin and the resulting change in the electrophoretic 
a)
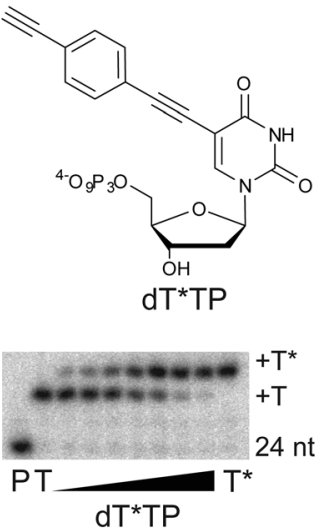

c)

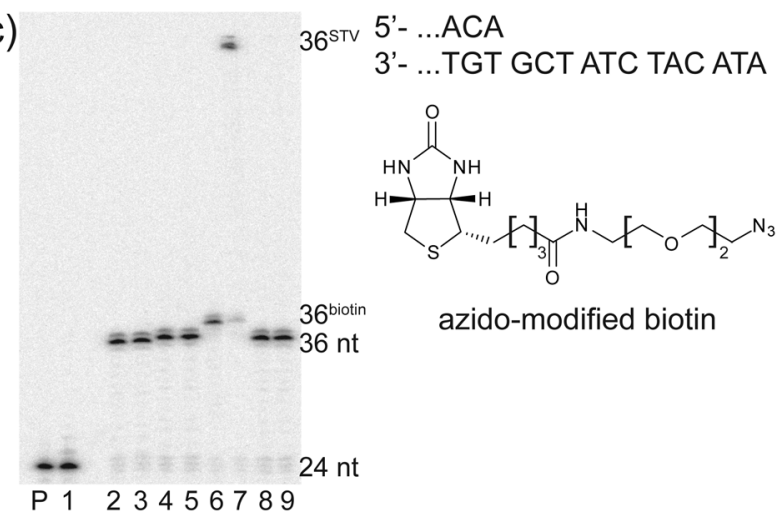

Fig. 1 (a) Structure of $\mathrm{dT}^{*} \mathrm{TP}$ and primer extension. A representative PAGE analysis of the competition experiment $\mathrm{dT}^{*} \mathrm{TP} v s$. dTTP using KlenTaq is shown. The ratio of $\mathrm{dT} * \mathrm{TP} / \mathrm{dTTP}$ was varied from $1 / 1$ to $100 / 1(1 / 1,2 / 1,4 / 1,10 / 1,20 / 1,50 / 1,100 / 1)$. (b) The same as in (a) for $\mathrm{dC}^{*} \mathrm{TP}$. (c) Primer extension using $\mathrm{dC}^{*} \mathrm{TP}$ followed by Click reaction. A partial sequence of the employed primer/template construct is indicated on the right. Lane P: primer only; lane 1: primer extension by KlenTaq in the presence of dATP, dGTP and dTTP; lane 2: all four natural dNTPs; lane 3: the same as lane 2 followed by incubation with streptavidin (STV); lane 4: the same as in lane 1, but in the presence of $\mathrm{dC} * \mathrm{TP}$; lane 5: the same as in lane 4 followed by incubation with STV; lane 6: the same as in lane 4 followed by Click reaction with azidomodified biotin; lane 7: the same as in lane 6 followed by incubation with STV; lane 8: the same as in lane 4 followed by incubation with azido-modified biotin (structure is shown) without BTTAA and $\mathrm{CuSO}_{4}$; lane 9: the same as in lane 8 followed by incubation with STV. For experimental details see ESI. $\dagger$

mobility revealed that the Click reaction product indeed carried biotin (Fig. 1c, lane 7).

To gain structural insight into how the modified pyrimidine analogs are processed by KlenTaq we crystallized KlenTaq bound to a primer/template complex and the modified $\mathrm{dT}^{*} \mathrm{TP}$ and $\mathrm{dC}^{*} \mathrm{TP}$ positioned at the dNTP binding site $\left(\operatorname{KlenTaq}\left(\mathrm{dT}^{*}\right)\right.$ or $\left.\operatorname{KlenTaq}\left(\mathrm{dC}^{*}\right)\right)$. To stall the respective modified substrates in the position poised for catalysis the polymerization reaction was terminated by the incorporation of ddCMP or ddGMP at the 3 '-primer terminus. The structures were solved by difference Fourier techniques and provide snapshots of the incorporation of the modified triphosphates at resolutions of $2.2 \AA$ and $1.8 \AA$, respectively (Fig. 2; Fig. S3, S4 and Table S1, ESI†).

In $\operatorname{KlenTaq}\left(\mathrm{dT}^{*}\right)$, the polymerase adopts a similar overall structure as it is observed for KlenTaq in complex with DNA and ddTTP (PDB: 1QTM; KlenTaq(1QTM)), resulting in a low r.m.s.d. for $\mathrm{C} \alpha$ of $0.417 \AA$. In analogy to KlenTaq(1QTM) the recognition of $\mathrm{dT}^{*} \mathrm{TP}$ relies on Watson-Crick base pairing and $\pi$-stacking interaction with the primer strand. Further all components required for efficient catalysis are properly arranged, except for two amino acids Arg660 and Arg587. Arg660 is positioned to make room for the rigid modification, thereby disabling interactions with the $3^{\prime}$-primer terminus, which are observed in the unmodified case (Fig. 2a and d). Similar effects were observed earlier in the case of another rigid non-polar modification that is processed about 2500 -fold less effective by KlenTaq. ${ }^{7 a}$ However, in the present study the incoming $\mathrm{dT}^{*} \mathrm{TP}$ is further stabilized by Arg587. The guanidinium group of Arg587 is located above the aromatic ring of the modification allowing cation $-\pi$ interaction (Fig. 2b). Further Arg587 stabilizes the phosphate backbone of the $3^{\prime}$-primer terminus. In addition, Lys663 is located on the other side of the aromatic ring also forming cation $-\pi$ interactions. Thus the aromatic ring is sandwiched between two positively charged amino acid side chains (Arg587 and Lys663). While the $\mathrm{C} 5$ modification at the nucleobase does not induce a conformational change of Lys663, it clearly affects the conformation of $\operatorname{Arg} 587$ (Fig. 2e). The distance from the primer $3^{\prime}$-terminus to the $\alpha$-phosphate of $\mathrm{dT}^{*} \mathrm{TP}$ is similar to the one observed in $\operatorname{KlenTaq}(1 \mathrm{QTM})$ indicating that $\mathrm{dT}^{*} \mathrm{TP}$ is properly aligned for catalysis (Fig. 2c and f). This interaction pattern may account for the proficient processing of $\mathrm{dT}^{*} \mathrm{TP}$ by KlenTaq.

The obtained structure of KlenTaq capturing dC*TP $\left(\operatorname{KlenTaq}\left(\mathrm{dC}^{*}\right)\right)$ shows very similar features as observed for KlenTaq (dT*) highlighted by the superimposed structures (Fig. S4, ESI $\dagger$ ). Once more, the incoming dC*TP is properly aligned and interacts via Watson-Crick base pairing with the templating $\mathrm{G}$. In analogy to KlenTaq(dT*), dC*TP is additionally stabilized by cation- $\pi$ interaction with Arg587 (Fig. S4b, ESI $\dagger$ ). The assembly of the catalytic residues with the required metal ions is very similar to $\operatorname{KlenTaq}\left(\mathrm{dT}^{*}\right)$ and consequently very similar to KlenTaq(1QTM) indicating that $\mathrm{dC}^{*} \mathrm{TP}$ is properly positioned (Fig. S4c, ESI $\dagger$ ).

In summary, the obtained structures of KlenTaq capturing modified substrates in the active site illustrate the plasticity of the enzyme and highlight the importance of the flexible regions of the dNTP binding pocket defined in the model of "active site tightness". ${ }^{13}$ Attaching the modification at the C5 atom of the pyrimidines has two major advantages. Firstly, modifications at this position cause minor disruption of the DNA duplex, since it points to the major groove. Secondly, they also result in minor disruption of the enzyme's ternary complex, since KlenTaq possesses sufficient plasticity to adapt to the structure of the modified substrates. This fact underpins the idea that the enzyme is able to respond to the incoming nucleotide by interacting with the modified substrates. Previous structural studies of flexible and polar modified substrates revealed hydrogen-bonding capability might improve their substrate properties. ${ }^{7}$ The present study focusing on rigid and non-polar modifications suggests that the introduction of an aromatic ring enables new interactions like cation- $\pi$ interaction with positively charged amino acid side chains like arginine or lysine. This fact might explain the efficient processing of $\mathrm{dT}^{*} \mathrm{TP}$ and $\mathrm{dC} * \mathrm{TP}$. For instance, the earlier employed 


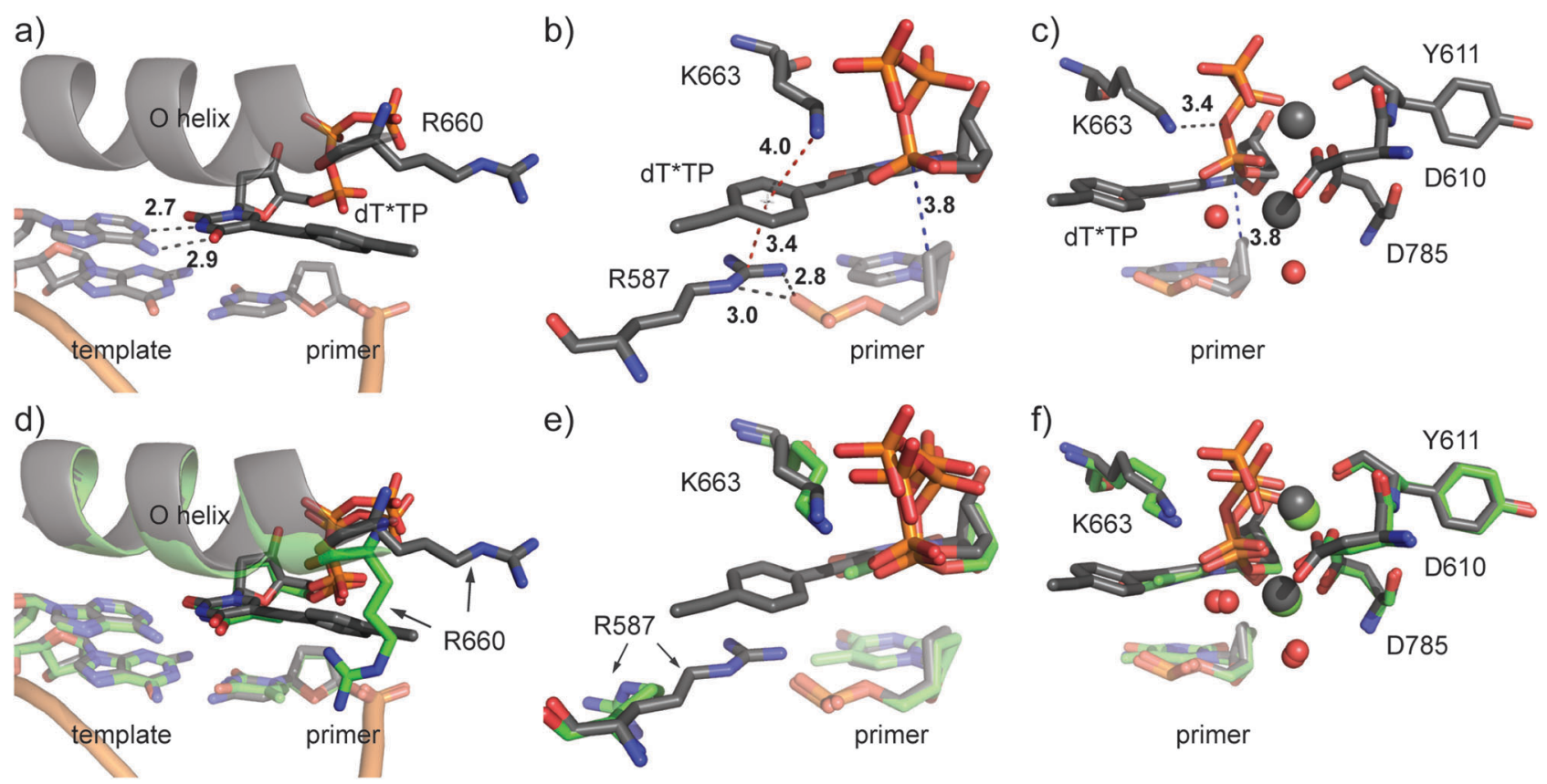

Fig. $2 K \operatorname{KlenTaq}\left(\mathrm{dT}^{*}\right)$ structure (gray). (a) Zoom into the active site of KlenTaq(dT*). The incoming dT*TP is stabilized via Watson-Crick base pairing. The $\mathrm{O}$ helix packs against the nascent base pair. R660 is indicated as stick. (b) The interaction pattern of the modified dT*TP with the protein side chains R587 and K663 (red dashed lines indicate cation- $\pi$ interactions). Distance of the $\alpha$-phosphate to the $3^{\prime}$-primer terminus is shown as a blue dashed line. Gray dashed lines indicate further interaction patterns. (c) Close-up view of the complexation of $\mathrm{Mg}^{2+}$ ions by the incoming $\mathrm{dT}^{*} \mathrm{TP}$ and the amino acids responsible for catalysis. (d)-(f) Shown are the same cut-outs and orientations as in (a)-(c) for KlenTaq(dT*) (gray) superimposed with KlenTaq(1QTM) (green). All distances are in Å.

thymidine analog ${ }^{7 a}$ that bears a rigid non-polar modification lacking an aromatic ring is less effectively processed as $\mathrm{dT}^{*} \mathrm{TP}$ (i.e. 7-versus 2500 -fold reduction of incorporation efficiency compared to dTTP). In line with these findings, Burgess and coworkers demonstrated that dyes attached to the nucleobase via a rigid conjugate linkage show enhanced spectroscopic properties and their substrate properties improve with the linker bearing an aromatic ring. ${ }^{14}$ These insights and the present study suggest that introduction of an aromatic ring at the discussed position in modified dNTPs may improve their substrate properties. This enhancement of design guidelines for the development of new modified dNTPs in combination with directed evolution of DNA polymerases ${ }^{15}$ will stimulate the development of future applications.

\section{Notes and references}

$\ddagger$ Data deposition: the atomic coordinates and structure factors have been deposited in the Protein Data Bank, www.pdb.org (PDB ID codes 4ELT and 4ELU).

1 (a) D. R. Bentley, et al., Nature, 2008, 456, 53; (b) J. Bowers, et al., Nat. Methods, 2009, 6, 593.

2 (a) H. Ruparel, L. Bi, Z. Li, X. Bai, D. H. Kim, N. J. Turro and J. Ju, Proc. Natl. Acad. Sci. U. S. A., 2005, 102, 5932; (b) T. D. Harris, et al., Science, 2008, 320, 106.

3 (a) J. Guo, et al., Proc. Natl. Acad. Sci. U. S. A., 2008, 105, 9145; (b) J. R. Pollack, C. M. Perou, A. A. Alizadeh, M. B. Eisen, A. Pergamenschikov, C. F. Williams, S. S. Jeffrey, D. Botstein and P. O. Brown, Nat. Genet., 1999, 23, 41; (c) A. Baccaro, A.-L. Steck and A. Marx, Angew. Chem., Int. Ed., 2012, 51, 254.

4 C. M. Perou, et al., Nature, 2000, 406, 747.

5 T. S. Seo, X. Bai, D. H. Kim, Q. Meng, S. Shi, H. Ruparel, Z. Li, N. J. Turro and J. Ju, Proc. Natl. Acad. Sci. U. S. A., 2005, 102, 5926.
6 (a) S. H. Weisbrod and A. Marx, Chem. Commun., 2007, 1828; (b) G. Mayer, Angew. Chem., Int. Ed., 2009, 48, 2672; (c) O. Thum, S. Jäger and M. Famulok, Angew. Chem., Int. Ed., 2001, 40, 3990; (d) H. Weizman and Y. Tor, J. Am. Chem. Soc., 2002, 124, 1568; (e) G. Giller, T. Tasara, B. Angerer, K. Mühlegger, M. Amacker and H. Winter, Nucleic Acids Res., 2003, 31, 2630; $(f)$ S. Jäger and M. Famulok, Angew. Chem., Int. Ed., 2004, 43, 3337; (g) J. P. Anderson, B. Angerer and L. A. Loeb, BioTechniques, 2005, 38, 257; (h) T. Ohbayashi, M. Kuwahara, M. Hasegawa, T. Kasamatsu, T. Tamura and H. Sawai, Org. Biomol. Chem., 2005, 3, 2463; (i) S. Jäger, G. Rasched, H. Kornreich-Leshem, M. Engeser, O. Thum and M. Famulok, J. Am. Chem. Soc., 2005, 127, 15071; (j) S. G. Srivatsan and Y. Tor, J. Am. Chem. Soc., 2007, 129, 2044; (k) P. Čapek, H. Cahová, R. Pohl, M. Hocek, C. Gloeckner and A. Marx, Chem.-Eur. J., 2007, 13, 6196; (l) P. M. E. Gramlich, C. T. Wirges, A. Manetto and T. Carell, Angew. Chem., Int. Ed., 2008, 47, 8350; $(m)$ M. Hocek and M. Fojta, Org. Biomol. Chem., 2008, 6, 2233; (n) S. Obeid, M. Yulikov, G. Jeschke and A. Marx, Angew. Chem., Int. Ed., 2008, 47, 6782.

7 (a) S. Obeid, A. Baccaro, W. Welte, K. Diederichs and A. Marx, Proc. Natl. Acad. Sci. U. S. A., 2010, 107, 21327; (b) K. Bergen, A. L. Steck, S. Strutt, A. Baccaro, W. Welte, K. Diederichs and A. Marx, J. Am. Chem. Soc., 2012, DOI: 10.1021/ja3017889 (available online).

8 A. H. El-Sagheer and T. Brown, Chem. Soc. Rev., 2010, 39, 1388 .

9 R. Chinchilla and C. Najera, Chem. Rev., 2007, 107, 874.

10 J. Gaster and A. Marx, Chem.-Eur. J., 2005, 11, 1861.

11 T. Kovács and L. Ötvös, Tetrahedron Lett., 1988, 29, 4525.

12 A. Baccaro and A. Marx, Chem.-Eur. J., 2010, 16, 218.

13 E. T. Kool, Annu. Rev. Biochem., 2002, 71, 191.

14 L. H. Thoresen, G.-S. Jiao, W. C. Haaland, M. L. Metzker and K. Burgess, Chem.-Eur. J., 2003, 9, 4603.

15 (a) A. M. Leconte, et al., Angew. Chem., Int. Ed., 2010, 49, 5921; (b) N. Ramsay, A.-S. Jemth, A. Brown, N. Crampton, P. Dear and P. Holliger, J. Am. Chem. Soc., 2010, 132, 5096; (c) N. Staiger and A. Marx, ChemBioChem, 2010, 11, 1963. 